\title{
Supplement to Estimating the Use of Public Lands: Integrated Modeling of Open Populations with Convolution Likelihood Ecological Abundance Regression
}

\author{
Lutz F. Gruber Erica F. Stuber Lyndsie S. Wszola Joseph J. Fontaine
}

This supplement refers to models presented in Section 4 of the main manuscript (Gruber et al., 2019).

\section{Model Validation}

Our Bayesian CLEAR analysis provides a posterior sample of the model parameters from which we can generate a posterior sample of the expected abundances $\lambda_{s, d}(t)$ and hunt durations $D_{s, d, t}$. We evaluate goodness of fit with a combination of scores and visual inspection of fitted vs. observed plots. AUC is used as a normalized and interpretable score for binary events classification; MSE as an unnormalized, but still interpretable score for fit, and log-likelihood (also known as the "log-score", Czado et al., 2009) as a non-interpretable, but proper scoring rule.

CLEAR is based on estimating the immigration and emigration functions. While the Hunter Survey did not directly observe immigration; exit interviews provide direct observations of realized emigration (hunt duration). To validate how well the estimated CLEAR model describes the realized population dynamics, we first check that emigration, in terms of hunt duration, is estimated well. Second, we check that the population point counts, which are realizations of both immigration and emigration, are estimated well. Together, these analyses imply that immigration is estimated well, too, which completes the model validation.

\section{Hunt duration}

Hunt duration $D_{s, d, t}$, as a direct realization of emigration, allows for direct validation of the estimates of that component process. Comparison of the posterior means with the realized values confirms that the emigration model describes the observed population dynamics suitably (Figure 1).

For added context, the estimates from CLEAR are compared to those of a frequentist Gamma GLM fitted to the interview data using the same training data and covariates (R function glm.fit with parameter family $=$ Gamma $($ link $=$ "log")). Post-estimation, the estimates from the GLM are truncated to the maximum possible hunt duration considering the censored sampling protocol, and the likelihood terms are adjusted accordingly (this model is referred to as the "capped" GLM below).

The best validation log-scores are obtained with CLEAR and prior variance 0.1, followed by CLEAR with prior variance 1, the capped frequentist GLM, CLEAR with prior variance 10, and the uncapped frequentist GLM. Ranking by MSE sees the capped GLM in the lead, while the rankings of the other models are consistent with the log-score ranking, see details in Table 1. 


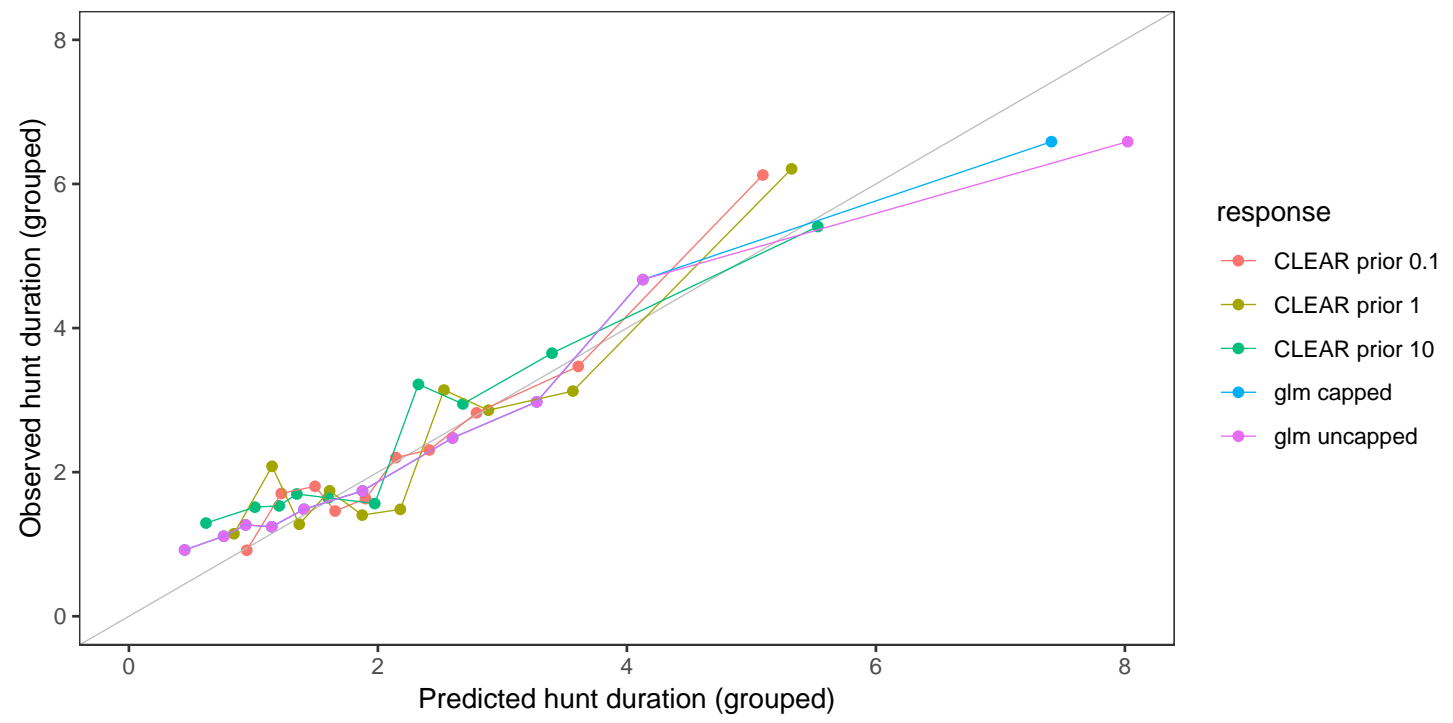

Figure 1: Comparison of posterior mean estimates (x-axis) and observed values (y-axis) of hunt duration in hours on the validation data. Individual data points are aggregated into 10 groups according to their estimated values, and the group means of the estimated and observed values are shown.

\begin{tabular}{lcc} 
Model & Log-score $\ell$ & MSE \\
\hline CLEAR, prior variance 0.1 & $\mathbf{2 9 7}$ & 4.11 \\
CLEAR, prior variance 1 & 286 & 4.21 \\
CLEAR, prior variance 10 & 268 & 4.57 \\
Gamma GLM uncapped & 265 & 4.96 \\
Gamma GLM capped & 273 & $\mathbf{3 . 3 2}$
\end{tabular}

Table 1: Log-score and MSE on the validation interview data for CLEAR with different priors and a frequentist GLM. 


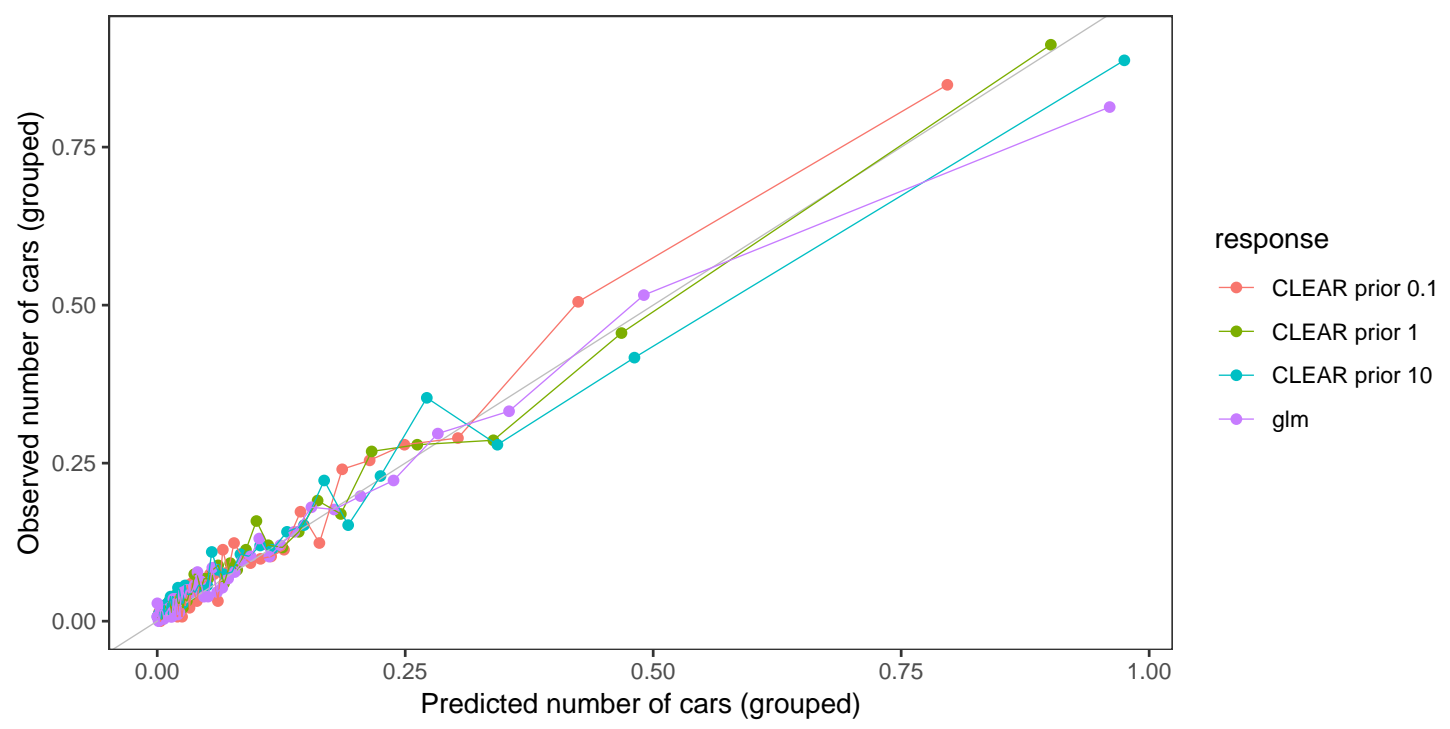

Figure 2: Comparison of posterior mean estimates of $\lambda_{s, d}(t)$ (x-axis) and observed values $N_{s, d}(t)$ (y-axis) on the validation data for low-frequency model. Individual data points are aggregated into 50 groups according to their estimated values, and the group means of the estimated and observed values are shown.

\begin{tabular}{lccc} 
Model & Log-score $\ell$ & MSE & AUC \\
\hline CLEAR, prior variance 0.1 & $-3,994$ & 0.211 & 0.780 \\
CLEAR, prior variance 1 & $\mathbf{- 3 , 9 6 3}$ & $\mathbf{0 . 2 0 9}$ & $\mathbf{0 . 7 8 7}$ \\
CLEAR, prior variance 10 & $-4,013$ & 0.211 & 0.782 \\
Gamma GLM & $-4,063$ & 0.213 & 0.786
\end{tabular}

Table 2: Log-score and MSE on the validation point count data for CLEAR with different priors and a frequentist GLM. The AUCs are for prediction of the binary event "at least one car present."

\section{Point counts}

Comparison of the posterior mean estimates of $\lambda_{s, d}(t)$ with the observed values $N_{s, d}(t)$ in the validation data confirms that the estimates are unbiased as well as contain a usable degree of differentiation (Figure 2).

To put the results of our CLEAR model in context, we compare the estimates from the low-frequency point count component model with estimates from a frequentist Poisson GLM fitted to just the low-frequency data using the same training data and covariates (R function glm.fit with parameter family = poisson (link $=" \log ")$ ). Note that the Poisson GLM analysis can not provide estimates of cumulative immigration over a time period, so it cannot be used in place of CLEAR analysis by itself; that said, it is a suitable benchmark for the low-frequency component model, which is also based on Poisson likelihoods, but whose location parameter is the result of the mechanistic population model and not a direct log-link regression.

Irrespective of the coefficient prior variance used, the CLEAR low-frequency point count component model outperforms the Poisson GLM in both mean squared error and log-score (see Table 2). The AUC score evaluates the predictive power of estimated abundance, which is not a probability estimate and not bounded from above by 1 , as a predictive score for the binary event "at least one car present." The CLEAR model with prior variance 1 has a slightly better AUC than the Poisson GLM, while the other CLEAR models have slightly lower AUC scores.

Furthermore, to illustrate the value of more recent data, compare the AUC scores of the high-frequency model (0.95-0.96, depending on the prior variance) with that of the low-frequency model (0.78-0.79). The 


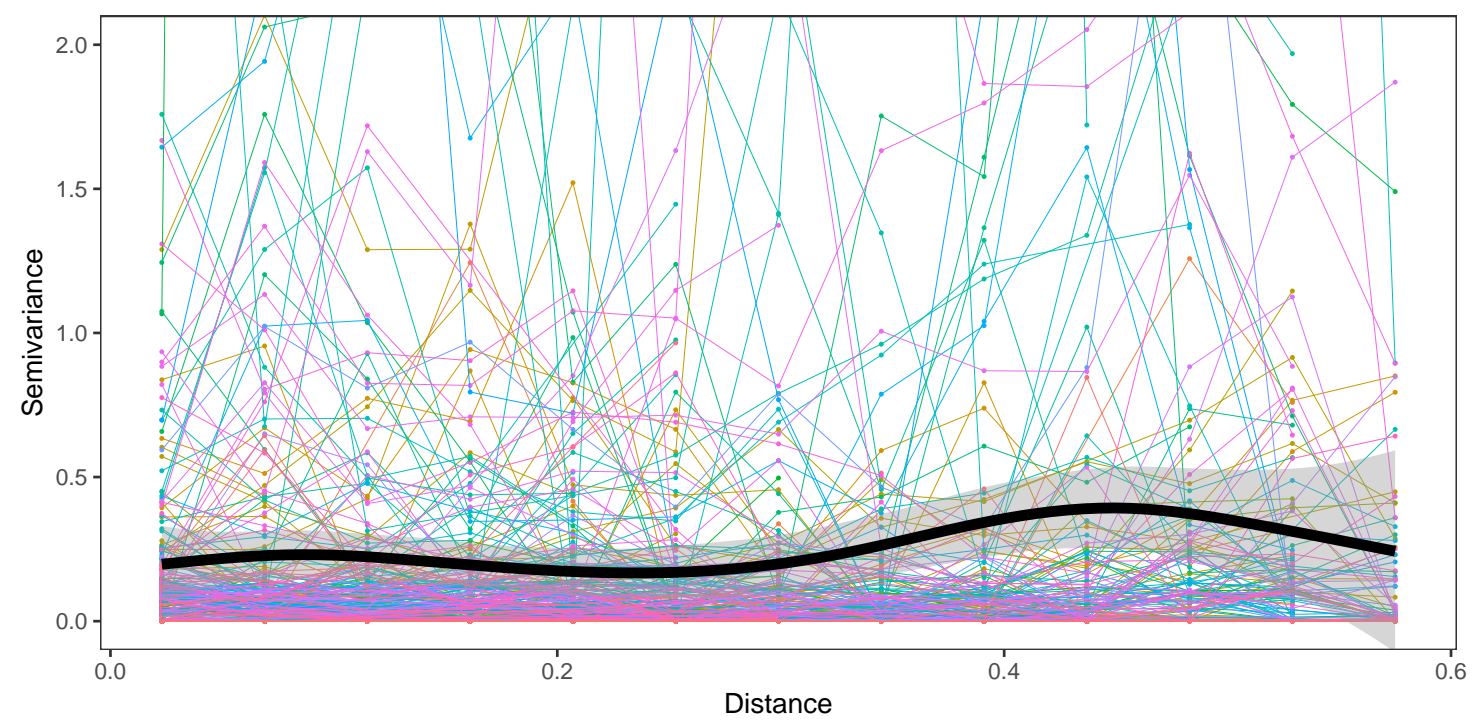

Figure 3: Variograms for the training data residuals of the low-frequency component model (colors indicate the different dates); the thick black line is a smoothed value across all dates and distances.

substantially higher AUC for the high-frequency component model is due to the observed counts from 15 minutes ago entering into the model as a predictor, while the low-frequency component model does not utilize previous counts as predictors.

A variogram analysis (Figure 3 confirms that the residuals of the low-frequency point count component model are free of undue spatial correlation. The residuals from the low-frequency and high-frequency point count models have only negligible temporal autocorrelation, with the median absolute first lag autocorrelation across all series being around 0.11 for the high-frequency model (observations are spaced 15 minutes) and less than 0.1 for the low-frequency model (observations are spaced 1 day); Figure 4 shows the median of the absolute values of the partial autocorrelations across all sites for lags 1 through 20. 

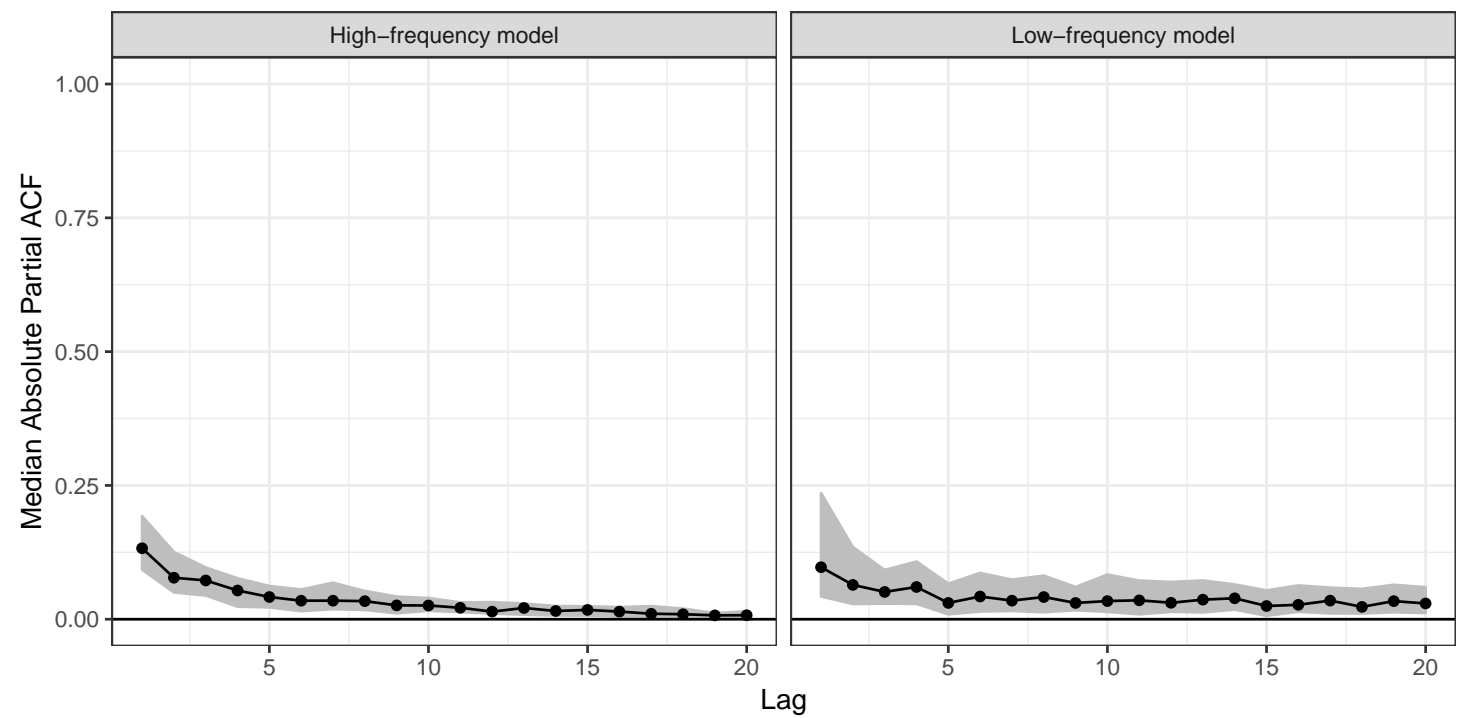

Figure 4: Black lines show the median of the absolute partial PACF values across all sites; the shaded areas show the interquartile range of the absolute PACFs.

\section{References}

Czado, C., Gneiting, T., and Held, L. (2009). "Predictive Model Assessment for Count Data." Biometrics, 65(4): 1254-1261.

URL https://onlinelibrary.wiley.com/doi/abs/10.1111/j.1541-0420.2009.01191.x 1

Gruber, L. F., Stuber, E. F., Wszola, L. S., and Fontaine, J. J. (2019). "Estimating the Use of Public Lands: Integrated Modeling of Open Populations with Convolution Likelihood Ecological Abundance Regression." Bayesian Analysis.

DOI https://doi.org/10.1214/19-BA1152 1 